\title{
Обновленная этиологическая
}

\section{классификация панкреатитов TIGAR-0 \\ (версия 2): адаптация для российской клинической практики}

Бордин Д.С., 2,3 • Кучерявый Ю.А., ${ }^{2,4}$ • Кирюкова М.А. ${ }^{1}$

Бордин Дмитрий Станиславович д-р мед. наук, заведующий отделом патологии поджелудочной железы, желчных путей и верхних отделов пищеварительного тракта'; профессор кафедры пропедевтики внутренних болезней и гастроэнтерологии ; профессор кафедры поликлинической терапии и семейной медицины факультета последипломного образованияз; ORCID: https://orcid. org/0000-0003-2815-3992.

Тел.: +7 (495) 30430 35, доб. 1585.

E-mail: d.bordin@mknc.ru

Кучерявый Юрий Александрович канд. мед. наук, доцент кафедры пропедевтики внутренних болезней и гастроэнтерологии; заведующий отделением гастроэнтерологии ${ }^{4}$; ORCID: https://orcid.org/0000-0001-7760-2091

$\triangle 143421$, Московская область, г.о. Красногорск, дер. Глухово, ул. Рублевское предместье, 2/2, Российская Федерация.

Тел.: +7 (495) 6453377

E-mail:proped@mail.ru

Кирюкова Мария Анатольевна -

мл. науч. сотр. отделения

патологии поджелудочной железы и желчевыводящих путей'; ORCID: https://orcid.org/0000-0002-6946-3826. E-mail:m.kirukova@mknc.ru
Открытия в молекулярной генетике и прорыв в технологиях визуализации, произошедшие за последние два десятилетия, перевернули наши представления о причинах и биомаркерах панкреатита, расширили знания о патогенезе заболевания и способствовали появлению новых дополнительных аспектов консервативного лечения. С практической точки зрения чрезвычайно важно, чтобы врач понимал этиологию болезни. Именно поэтому уже продолжительное время идет работа по созданию этиологических классификаций панкреатита. Первой международно признанной системой стала TIGAR-O, увидевшая свет в 2001 г. Будучи абсолютно инновационной, она структурировала представления об этиологии хронического панкреатита. Вторая версия (редакция) этой системы опубликована в 2019 г. и мало знакома отечественному читателю. При этом, на наш взгляд, документ существенно дополнен и выстроен таким образом, чтобы быть максимально удобным и полезным практикующему врачу. Данная обзорная статья содержит ключевые положения второй версии TIGAR-O и рекомендации по их адаптации к российской клинической практике.

Ключевые слова: хронический панкреатит, острый панкреатит, классификация, диагностика, генетические факторы, внешнесекреторная недостаточность поджелудочной железы, сахарный диабет

Для цитирования: Бордин ДС, Кучерявый ЮА, Кирюкова МА. Обновленная этиологическая классификация панкреатитов TIGAR-O (версия 2): адаптация для российской клинической практики. Альманах клинической медицины. 2020;48(6):34963. doi: 10.18786/2072-0505-2020-48-062.

Поступила 19.08.2020; доработана 17.11.2020; принята к публикации 19.11.2020; опубликована онлайн 10.12.2020

'ГБУЗ г. Москвы «Московский клинический научно-практический центр имени А.С. Логинова ДЗМ»; 111123,

г. Москва, шоссе Энтузиастов, 86, Российская Федерация

2 ФГБОУ ВО «Московский государственный медико-стоматологический университет имени А.И. Евдокимова» Минздрава России; 127473, г. Москва, ул. Делегатская, 20-1, Российская Федерация

${ }^{3}$ ФГБОУ ВО «Тверской государственный медицинский университет» Минздрава России; 170100, г. Тверь, ул. Советская, 4, Российская Федерация

${ }^{4} \mathrm{AO}$ «Ильинская больница»; 143421, Московская область, г.о. Красногорск, дер. Глухово, ул. Рублевское предместье, 2/2, Российская Федерация 
$\Pi$

ричины острого панкреатита (ОП), рецидивирующего ОП (РОП) и хронического панкреатита (ХП) могут быть идентичными для разных фенотипов воспалительных заболеваний поджелудочной железы (ПЖ). Вариативные комбинации генетических, эпигенетических, метаболических факторов и факторов окружающей среды, по-видимому обладая кумулятивным эффектом, инициируют и стимулируют воспалительный процесс и его последствия в нескольких системах, обычно регулирующих и поддерживающих функционирование ПЖ [1]. Взаимосвязь между фенотипами воспалительных заболеваний ПЖ подчеркивается доказанными рисками трансформации одной формы панкреатита в другую. Так, ОП переходит в РОП примерно в 20\% случаев, РОП в ХП - в $35 \%$, в то время как у $40 \%$ пациентов с ХП в анамнезе нет ОП или РОП и паттерны прогрессии определяются множественными факторами риска и патогенетическими механизмами [2].

Как известно, этиотропный подход наиболее целесообразен и, в конечном итоге, эффективен для предотвращения тяжелого течения заболевания и модификации тактики ведения больного с панкреатитом [3]. Именно поэтому важно пытаться определить этиологию панкреатита и оценить значение всех факторов риска в дебюте заболевания, что позволит персонифицированно сформировать представление о прогнозе и потенциальной профилактике РОП и ХП, а также их осложнений - рефрактерной абдоминальной боли, экзокринной недостаточности поджелудочной железы (ЭНПЖ), сахарного диабета (СД) и рака поджелудочной железы (РПЖ) [1]. Большинство этиологических факторов панкреатита и механизмов патогенеза к настоящему времени хорошо изучено, что позволяет составлять эффективные терапевтические планы в соответствии с принципами доказательной медицины [4].

Предшествующая версия этиологической классификации TIGAR-O (версия 1) опубликована в 2001 г. [5], ее название представляет собой аббревиатуру и включает первые буквы шести основополагающих факторов риска или этиологических факторов развития панкреатита:

- токсико-метаболический (Т);

- идиопатический (I);

- генетический $(\mathrm{G})$;

- аутоиммунный (A);

- рецидивирующий (R) и тяжелый ОП;

- обструктивный (O).

Последняя категория была отделена от других дефисом для обособления этиологии повреждения ПЖ экстраацинарной природы. Система предназначалась для регистрации каждого из возможных факторов риска (препанкреатит) или определенных этиологических факторов, способствующих его развитию (постпанкреатит), на основе нового механистического подхода, заключающегося в обратном проектировании патогенеза сложных заболеваний [6]. Первая версия TIGAR-O показала свою состоятельность в клинической практике, множестве клинических и эпидемиологических исследований, консенсусных документах в разных регионах мира, включая Россию [7-9]. Следующей исторически значимой этиологической классификацией ХП, увидевшей свет в 2007 г., стала система M-ANNHEIM [10], которая включала систему классификации тяжести $\mathrm{XП} \mathrm{[11].}$

В исследованиях последних двух десятилетий получено много новой информации о факторах риска панкреатита и механизмах его развития. Принципиально важно рассмотреть данные, касающиеся количественного употребления алкоголя в контексте восприимчивости и потенциального прогрессирования заболевания, независимой роли курения, значения гипертриглицеридемии (ГТГ), подтипов аутоиммунного панкреатита (АИП), ряда генетических аспектов, и новые представления о влиянии сложных генотипов, особенностей патофизиологии ПЖ при обструктивных панкреатитах, СД и РПЖ. Все это стало основой для обновления перечня факторов риска/этиологии панкреатитов в рамках второй версии TIGAR-O V2 [1].

\section{Структура TIGAR-O V2}

Вторая версия разработана на базе TIGAR-O 2001 г. [5] и существенно дополнена (таблица) по иерархической структуре. Она обеспечивает бо́льшую специфичность и точность в отношении факторов риска/этиологии у пациента. Отмечено, что некоторые агенты служат первичными факторами повреждения или стресса, другие повышают восприимчивость за счет снижения толерантности к повреждению или стрессу, а третьи влияют на защитные реакции, подавляют регенерацию или вносят свой вклад в патогенез другими способами. Каждая из шести категорий и некоторых подкатегорий включает в себя раздел «неуточненный» (англ. not otherwise specified, NOS), в который можно добавлять другие, в том числе нетипичные факторы. Таким образом, чеклист пациента представляет собой список факторов, связанных с развитием панкреатита, который должен дополняться и датироваться по мере 
Классификация факторов риска и этиологии панкреатитов TIGAR-O V2 (адаптировано по [1])

Этиологические факторы риска / подтип

Токсические/метаболические факторы
Характеристики и комментарии
Алкоголь

Курение

Гиперкальциемия

Гипертриглицеридемия

Медикаменты

Другие токсические факторы

Факторы, связанные с окислительным стрессом

Метаболические факторы

\begin{abstract}
Идиопатические факторы
\end{abstract}
Раннее начало

Позднее начало

Генетические факторы

Предполагаемый наследственный ХП

Аутосомно-доминантные формы ХП

Аутосомно-рецессивные формы ХП
Количество употребляемого алкоголя:

- от 0 до < 1 стандартной дозы алкоголя в день (включая не употребляющих алкоголь и употребляющих редко)

- 1-2 стандартные дозы алкоголя в день

- 3-4 стандартные дозы алкоголя в день

- 5 или более стандартных доз алкоголя в день

Оценивается:

- восприимчивость (до верификации панкреатита)

- прогрессирование (после верификации панкреатита)

Если да, указать индекс «пачко-лет»

Не курит (< 100 сигарет за всю жизнь)

Курил в прошлом

Курит

Другое

Уровень общего кальция > 12 мг/дл или 3 ммоль/л

Риск гипертриглицеридемии (натощак > 300 мг/дл; после еды > 500 мг/дл)

ОП на фоне гипертриглицеридемии в анамнезе (> 500 мг/дл в первые 72 часа)

Семейная гипертриглицеридемия

См. текст статьи

ХБП (5-я стадия - терминальная ХБП):

- на диализе

- без диализа

Перенес трансплантацию почки

Лучевая терапия/химиотерапия

Ишемический панкреатит

Другие токсины

Сахарный диабет (с датой диагностики, если известна):

- контролируется диетой

- необходимы пероральные препараты

- инсулинпотребный СД ( $\geq 10$ ед/д или $\geq 0,1$ ед/кг/д)

Рацион питания (красное мясо < 60 г/сут, вегетарианская/веганская диета)

Ожирение (ИМТ > 30 кг/м²)

Висцеральное ожирение

Другое

$<35$ лет

$\geq 35$ лет

Нет результатов или ограниченные данные генетического исследования при наличии признаков наследственного характера поражения Пж

Мутация гена PRSS1 (наследственный панкреатит)

Мутация гена CEL (фенотип MODY8)

Мутации гена CFTR, 2 тяжелых варианта в транс-положении (муковисцидоз)

Мутации гена CFTR, <2 тяжелых вариантов в транс-положении (CFTR-ассоциированное заболевание)

$\geq 1$ мутации CFTR (все в цис-положении) при положительном потовом тесте и поражении хотя бы одного органа-

мишени (CFTR- ассоциированное заболевание)

Мутации SPINK1, 2 патогенных варианта в транс-положении (SPINK1-ассоциированный семейный панкреатит)

Другое 
Варианты ХП со сложным наследованием

\begin{abstract}
Гены-модификаторы
\end{abstract}
Семейная гипертриглицеридемия

Редкие неопухолевые генетически обусловленные нарушения Пж $\geq 1$ варианта гена CFTR (все в цис-положении) при сомнительных результатах потового теста либо при отсутствии его результатов

Варианты гена CTRC

Варианты гена CASR

$\geq 1$ варианта гена SPINK1 (все в цис-положении)

Варианты гена CPA1

Варианты гена CEL или CEL-HYB

Другое

PRSS1-PRSS1 локус

$C L D N 2$ локус

SLC26A9

GGT1

ABO - группа крови B (III)

Другое

Дефицит липопротеинлипазы LPL

Дефицит аполипопротеина C II APOC2

Другие синдромы семейной хиломикронемии

Многофакторный синдром хиломикронемии

Другое

Синдром Швахмана - Даймонда

Синдром Йохансона - Близзарда

Митохондриальные заболевания

Другое

АИП/панкреатит, отвечающий на лечение кортикостероидными препаратами

$$
\begin{aligned}
& \text { АИП 1-го типа - IgG4-ассоциированное } \\
& \text { заболевание }
\end{aligned}
$$

АИП 2-го типа

АИП неуточненный

РОП и тяжелый ОП

ОП

ОП внепанкреатической этиологии
Поражена изолированно Пж

Поражены другие органы (lgG4-ассоциированная болезнь)

Поражена изолированно Пж

Сочетается с болезнью Крона

Сочетается с язвенным колитом

Поражены другие органы

Отвечающий на стероиды, но не 1-й и не 2-й тип

Единственный эпизод:

• ОП без персистирующей полиорганной недостаточности и < 30\% панкреонекроза

- ОП без персистирующей полиорганной недостаточности и > 30\% панкреонекроза

- ОП с персистирующей полиорганной недостаточностью и < 30\% панкреонекроза

• ОП с персистирующей полиорганной недостаточностью и $\geq 30 \%$ панкреонекроза

Билиарный панкреатит

После ЭРХПГ

Травматический

Ишемический

Инфекционный: вирусный, другой (не вторичная инфекция)

Неопределенный/неуточненный

Исключены алкогольная этиология, гипертриглицеридемия, гиперкальциемия и генетическая этиология

Обструктивные факторы

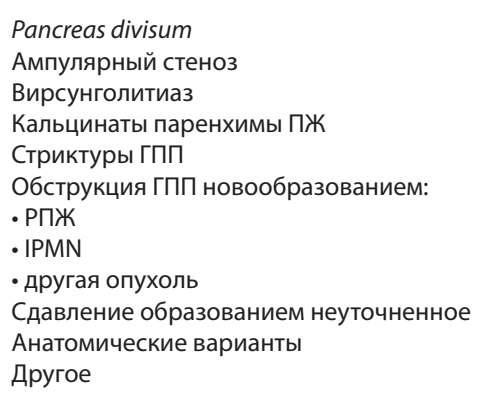

IPMN - intraductal papillary mucinous neoplasm (внутрипротоковые папиллярно-муцинозные неоплазии), АИП - аутоиммунный панкреатит, ГПП - главный панкреатический проток, ИМТ - индекс массы тела, ОП - острый панкреатит, ПЖ - поджелудочная железа, РОП - рецидивирующий острый панкреатит, РПж - рак поджелудочной железы, СД - сахарный диабет, ХБП - хроническая болезнь почек, ХП - хронический панкреатит, ЭРХПГ - эндоскопическая ретроградная холангиопанкреатография 
получения новой информации. Такой подход обеспечивает более точное понимание патогенеза и течения заболевания во времени у конкретного пациента и дает возможность оценивать эффективность лечения [1].

\section{Токсико-метаболические факторы}

Группа токсико-метаболических факторов фокусируется на агентах, которые специфически вызывают нарушение функции, или повреждение ацинарных/протоковых клеток, или изменяют реакцию клеток, связанных с ПЖ в цепочке «повреждение $\rightarrow$ воспаление $\rightarrow$ разрешение $\rightarrow$ регенерация и ее последствия» [12].

\section{Алкоголь}

ХП, связанный с употреблением алкоголя, на основании ряда исследований стратифицирован по уровням:

1) от 0 до < 1 стандартной дозы алкоголя в день или время от времени;

2) 1-2 стандартные дозы алкоголя в день;

3) 3-4 стандартные дозы алкоголя в день;

4) 5 или более стандартных доз алкоголя в день.

Риск ХП существенно возрастает при употреблении приблизительно 4-5 и более стандартных доз алкоголя в день (стандартная доза алкоголя $=13,7$ мл чистого спирта), особенно при запойном алкоголизме или длительном употреблении алкоголя. После первого эпизода ОП продолжительное употребление алкоголя с дозозависимым эффектом увеличивает риск РОП, а также скорость прогрессирования до ХП и развития СД и других осложнений [13]. Безопасный уровень употребления алкоголя после эпизода ОП и особенно РОП без риска прогрессирования еще не определен. У многих пациентов ОП, РОП и ХП развиваются при употреблении алкоголя ниже ожидаемого порогового уровня в случае тяжелого алкоголизма. Периодическое употребление алкоголя или употребление алкоголя в компании (англ. social drinking) довольно распространено среди взрослых пациентов, однако панкреатит у них встречается редко - даже среди пациентов, употребляющих алкоголь в больших количествах. Кроме того, во многих популяциях, включая США, у большинства пациентов с РОП и ХП этиология не связана с алкоголем, что указывает на то, что для развития ОП, РОП и ХП необходимы дополнительные факторы или случайные инициирующие события (генетическая предрасположенность и воздействие факторов внешней среды) [1].
Курение

Курение выступает независимым фактором риска развития РОП и ХП, причем продолжающие курить пациенты подвержены более высокому риску, чем отказавшиеся от этой вредной привычки. Риск от курения возрастает с увеличением количества выкуриваемых сигарет в день [3]. Для регистрации данных о курении сигар, трубок или марихуаны может использоваться раздел «Другое». Категория «курил в прошлом» предназначена для описания пациентов, ранее подвергавшихся воздействию курения, что могло инициировать процесс развития панкреатита. Категория «продолжает курить» предназначена для пациентов как куривших в прошлом, так и продолжающих курить. Такой подход описывает паттерн и степень воздействия и может использоваться для расчета «пачко-лет» (количество пачек в день $\times$ годы курения) [1].

\section{Гиперкальичемия}

В качестве фактора риска развития ОП и ХП гиперкальциемия рассматривается, когда уровень общего кальция крови составляет $\geq 12,0$ мг/дл ( $\geq 3$ ммоль/л). Примерно 90\% случаев гиперкальциемии вызваны первичным гиперпаратиреозом (ПГПТ) или гиперкальциемией на фоне злокачественных новообразований, и лишь небольшая доля случаев ассоциирована с генетическими нарушениями, саркоидозом, хронической болезнью почек (ХБП) и другими причинами.

Гиперпаратиреоз вызывает гиперкальциемию, обычно сопровождающуюся гипофосфатемией, но ОП среди больных с ПГПТ возникает менее чем в 7\% случаев. Риск ОП коррелирует с самыми высокими уровнями кальция в сыворотке крови среди пациентов с ПГПТ [14]. Семейная гипокальциурическая гиперкальциемия - аутосомно-доминантное заболевание, связанное со специфическими мутациями в гене плейотропного кальцийчувствительного рецептора (англ. calcium-sensing receptor gene, CASR), отвечающего за различные реакции в клетках. Самостоятельно мутации CASR не вызывают ОП, РОП и ХП, но есть данные о случаях диагностики панкреатита у пациентов с ПГПТ и мутациями CASR. Следовательно, комплексный риск панкреатита, связанный с генетическими вариантами CASR, может иметь место и в отсутствие гиперкальциемии [15]. Категория «Другое» предназначена для выявленных причин гиперкальциемии, таких как опухоли паращитовидных желез, множественная эндокринная неоплазия типа 1 или $2 \mathrm{a}$, 
множественная миелома или иные редкие причины [1].

Дополнительно следует учитывать такие факторы, как мочекаменная болезнь, прием лекарственных препаратов, в том числе препаратов лития и витамина $\mathrm{D}$, а также основные лабораторные показатели, включая уровни паратиреоидного гормона, общего кальция и фосфора в сыворотке, уровень кальция в моче, если подозревается семейная гипокальциурическая гиперкальциемия.

\section{Гипертриглииеридемия}

Неоднозначные данные ранних исследований роли гиперлипидемии в развитии ХП стали причиной того, что в качестве фактора риска вместо гиперлипидемии стали рассматривать гипертриглицеридемию. Продолжаются споры о том, какой уровень триглицеридов (ТГ) считать диагностическим - минимальный (натощак) или максимальный (во время болевого эпизода панкреатита или после приема пищи), поскольку риск ОП увеличивают именно постпрандиальные значения ТГ. Так, риск ОП возрастает при легком и умеренном повышении ТГ (>177 мг/дл) в крови после еды (отношение рисков (ОР) 2,3 для уровня ТГ 177265 мг/дл) и коррелирует с ростом постпрандиальной ГТГ (ОР 2,9 для уровня ТГ 353-366 мг/дл, ОР 3,9 для уровня ТГ 354-442 мг/дл и ОР 8,7 для уровня ТГ > 442 мг/дл) [15]. Важно отметить, что ГТГ не только увеличивает риск развития ОП, РОП и ХП, но также усиливает тяжесть эпизода ОП в отношении ряда параметров, в том числе развития панкреонекроза, синдрома системной воспалительной реакции, полиорганной недостаточности, госпитализации в отделение реанимации и интенсивной терапии, длительности пребывания в стационаре и смертности [16].

\section{Лекарственные препараты}

Риск развития ОП и ХП может повышаться под воздействием более 100 лекарственных средств, включая метилпреднизолон, фенофибрат, ингибиторы ангиотензинпревращающего фермента, статины, эстрогены, вальпроевую кислоту, с наибольшей доказательностью - азатиоприн (и его метаболит 6-меркаптопурин), 2',3'-дидезоксиинозин и L-аспарагиназу. Механизмы панкреотоксического действия могут быть различными, при этом прямые причинно-следственные связи обычно отсутствуют. В большинстве описанных клинических наблюдений не были исключены сопутствующие факторы, такие как, например, генетические [17]. Именно поэтому в TIGAR-O V2 не содержится конкретного списка лекарственных препаратов с потенциальным панкреотоксическим действием [1].

\section{Токсинь}

Под токсинами подразумевается любой агент, потенциально вызывающий повреждение ацинарных или протоковых клеток ПЖ, за исключением алкоголя и табака, в том числе ХБП, факторы, вызывающие окислительный стресс, и другие токсические вещества.

Хроническая болезнь почек. В первой версии TIGAR-O использовался термин «хроническая почечная недостаточность». B TIGAR-O V2 терминология обновилась, и степень тяжести ХБП определяется как стадия 5 или терминальная стадия хронической почечной недостаточности со скоростью клубочковой фильтрации менее 15 мл/мин или ниже вне зависимости от того, находится ли пациент на диализе или же он перенес трансплантацию почек. ХБП может утяжелять течение ОП или ХП вследствие либо невозможности элиминировать метаболиты обмена ПЖ либо дисбаланса электролитов, как, например, при гиперкальциемии. У пациентов с ХБП также возникают трудности в коррекции водно-электролитных нарушений и оценке эпизодов ОП [1].

Факторы, связанные с окислительным стрессом. Эти факторы включены в отдельную категорию на основании полученных в некоторых исследованиях данных об определенной эффективности антиоксидантов. Кроме того, в этот подраздел отнесены панкреатиты, ассоциированные с лучевой и химиотерапией, хронической ишемией, а также с любыми факторами, вызывающими токсичность через механизмы окислительного стресса, действие которых может быть смягчено антиоксидантами. Изолированное воздействие данных факторов недостаточно для того, чтобы вызывать панкреатит, но они, вероятно, вносят свой вклад в его развитие на фоне употребления алкоголя, курения, мальабсорбции или дефицита в рационе витаминов и антиоксидантов, изнурительных физических упражнений и/или наличия генетических факторов [1].

Подраздел «Другое» в категории «Токсины» включает в себя агенты с дозозависимым или идиосинкразийным действием, которые вызывают ОП, РОП или ХП за счет гиперстимуляции (например, яд скорпиона и антихолинэстеразные инсектициды) [18]. В этой подкатегории можно также указать факторы, которые считаются 
токсичными и повышают риск, но имеют неизученный механизм действия.

\section{Метаболические и другие факторь}

Это новая категория, объединяющая СД, ожирение, метаболический синдром, рацион питания и другие факторы, вероятно, связанные с развитием РОП и/или ХП, при этом ГТГ выделена выше в отдельную категорию из-за уникального механизма прямой токсичности жирных кислот, повышающей заболеваемость и риск осложнений при РОП и ХП $[1,16]$.

Сахарный диабет может быть причиной атрофии и фиброза ПЖ (то есть экзокринной панкреатопатии), осложнением ОП и ХП, а также выступать ранним диагностическим маркером РПЖ. Панкреатогенный сахарный диабет (тип 3c) в ряде случаев из-за потери островковых a-клеток, продуцирующих глюкагон, характеризуется лабильным течением, определяя склонность к тяжелой гипогликемии [19]. Во многих случаях сложно ответить на вопрос, наступил диабет до или после инициации воспаления, затронувшего экзокринный аппарат ПЖ, но цель состоит лишь в том, чтобы зафиксировать наличие и степень тяжести эндокринной недостаточности. О наличии СД уместно говорить при повышении уровня гликолизированного гемоглобина $\geq 6,5 \%$, глюкозы в плазме натощак $\geq 126$ мг/дл или уровня глюкозы $\geq 200$ мг/дл через 2 часа в рамках глюкозотолерантного теста с 75 г глюкозы. Термин «предиабет» используется для описания состояния, при котором уровень гликолизированного гемоглобина или глюкозы в сыворотке крови выходит за пределы нормальных, но не является диагностически значимым для СД, например, уровень гликолизированного гемоглобина 5,7-6,4\% и глюкозы в плазме натощак 100-125 мг/дл или 140-199 мг/дл после глюкозотолерантного теста. Если результаты исследований не дают однозначного ответа, их следует повторить [1].

Рацион питания. Диета может быть важным фактором, меняющим течение заболевания прямо или косвенно, например, путем воздействия на микробиом кишечника. Рацион питания пациента или диета выступают одним из самых сложных для оценки факторов. Порог риска РОП и ХП был обнаружен при потреблении красного мяса в количестве более чем 24,5 г/1000 ккал/сут (ОР 1,37, 95\% доверительный интервал 1,01-1,87), при этом интересно, что он не растет с увеличением количества красного мяса в рационе [20]. Около 60 г красного мяса пороговый уровень для рациона в 2300 ккал/день, поэтому «2 унции» ( 57 г) были произвольно приняты в качестве обоснованного порогового уровня риска для большинства взрослых. В свете вышесказанного авторы указывают, что вегетарианская / веганская диета должна рассматриваться как возможный защитный фактор [1].

Ожирение. Висцеральное ожирение, то есть избыток висцеральной жировой ткани, связано с риском развития ГТГ, СД, метаболического синдрома и других заболеваний [21], включая заболевания ПЖ [22]. На висцеральное ожирение указывает отношение талии к бедрам >0,9 у мужчин и > 0,85 у женщин. Общепринятые высокие значения индекса массы тела, характерные для ожирения, не обязательно исключают мальнутрицию у пациентов с заболеваниями ПЖ. Пациенты с высоким потреблением простых углеводов или низким содержанием жиров в рационе могут иметь избыточный вес или ожирение и при этом не проявлять симптомов мальдигестии, но в то же время иметь дефицит белка и/или микроэлементов, прежде всего жирорастворимых витаминов и витамина $\mathrm{B}_{12}$. Таким образом, высокий индекс массы тела не должен быть причиной отказа от проведения полного анализа нутритивного статуса пациента $[1,3,7,23]$.

\section{Идиопатический панкреатит}

B первой версии TIGAR-O идиопатический панкреатит подразделяли на 3 подтипа - с ранним и поздним манифестом и тропический. B TIGAR-O V2 категории раннего и позднего начала панкреатита являются основными и дифференцируются по возрасту <35 и $\geq 35$ лет [1]. Тропический панкреатит, по-видимому, представляет собой сложное генетически обусловленное заболевание, он обсуждается далее в разделе «Редкие неопухолевые генетически обусловленные нарушения поджелудочной железы».

\section{Наследственный панкреатит}

За последние 20 лет был достигнут существенный прогресс в понимании генетических аспектов панкреатита, при этом в большинстве случаев идиопатического и алкогольного панкреатита описаны важные генетические факторы, оказывающие влияние на развитие заболевания [24]. B TIGAR-O V2 приведено восемь генетических категорий.

Предполагаемый наследственный хронический панкреатит

Данная форма устанавливается при подозрении на наличие генетических факторов, во время 
ожидания результатов генетического тестирования, либо когда первоначальный генетический тест содержал ограниченное число параметров (например, только исследование частых мутаций в генах PRSS1, CFTR, SPINK1 и CTRC). Генетическая этиология должна рассматриваться в следующих случаях [1]:

- $\quad$ ранний возраст развития ХП (моложе 35 лет);

- идиопатический ОП, РОП и ХП, когда отсутствуют другие очевидные причины (такие как желчнокаменная болезнь или тяжелая абдоминальная травма);

- наследственный анамнез отягощен по ХП, СД, дислипидемии и РПЖ;

- имеются необычные биомаркеры, позволяющие предположить наследственную этиологию заболевания (например, в случае муковисцидоза);

- при условии тяжелого прогредиентного течения заболевания или плохого ответа на проводимое лечение.

\section{Аутосомно-доминантный панкреатит}

В эту категорию включены синдромы с менделевским наследованием, в том числе панкреатит, обусловленный мутациями приобретения функции (англ. gain of-function) в гене PRSS1, и фенотип MODY8, связанный с вариантами гена CEL.

\section{Аутосомно-рецессивные заболевания}

Аутосомно-рецессивные заболевания ПЖ с менделевским наследованием включают в себя классическую форму муковисцидоза, CFTRассоциированные заболевания и биаллельные патогенные мутации гена SPINK1.

Муковисиицоз и CFTR-ассоииированные заболевания. Прежде всего стоит отметить, что все мутации гена CFTR в зависимости от своего эффекта на белок-продукт гена подразделяются на 7 классов (I-VII) [25]. Мутации классов I, II, III и VII считаются тяжелыми. У пациентов с двумя патогенными вариантами CFTR этих классов, затрагивающими обе копии гена, то есть находящимися в транс-положении, как правило, развивается клиническая картина классической формы аутосомно-рецессивного заболевания муковисцидоз, которая включает в себя типичные для муковисцидоза жалобы и симптомы со стороны более чем одного органа-мишени и сопровождается нарушением функции белка CFTR. Последнее может быть выявлено путем проведения потовой пробы [26]. В то же время при обнаружении у пациента наряду с тяжелыми мутациями I, II, III и VII классов мутаций гена CFTR классов IV, V или VI в транс-положении относительно друг друга также может быть установлен диагноз муковисцидоза. Это возможно, если указанный генотип пациента сочетается с наличием у него клинических симптомов, характерных для муковисцидоза, даже если патологический процесс затрагивает лишь один орган-мишень. При этом для установления диагноза муковисцидоза в таком случае также необходимо подтвердить заболевание результатами потовой пробы [27].

В некоторых случаях доминирующим признаком заболевания у пациентов с мутациями гена CFTR становится панкреатит. Состояния, которые не могут быть определены как муковисцидоз (например, моносимптомные - затрагивающие только один орган, например, ПЖ, и имеющие 1 патогенный вариант CFTR любого класса), классифицируются как CFTRассоциированные заболевания [1]. Как правило, это ситуации, когда у больного патологическим процессом затронут лишь один орган, например, ПЖ, то есть наблюдается моносимптомное течение заболевания, потовый тест положителен или дает сомнительный результат (обычно хлориды пота лежат в диапазоне 30-59 ммоль/л), a в генотипе выявлена лишь одна мутация CFTR любого класса, либо больной - носитель генного варианта CFTR неопределенного значения. Отметим: CFTR-ассоциированные заболевания по сути нельзя отнести к категории аутосомно-рецессивных болезней, так как они связаны с наличием генного дефекта только в одной копии гена CFTR. Однако не исключено, что второй вариант гена CFTR у таких пациентов просто еще не идентифицирован в силу ограничения применяемых методик генотипирования или недостаточности знаний в отношении молекулярных особенностей функционирования CFTR на данный момент. Тем не менее категория CFTR-ассоциированных заболеваний крайне важна, поскольку она может влиять на выбор терапевтической тактики ведения пациента. Кроме панкреатита к CFTR-ассоциированным заболеваниям относят мужской фактор бесплодия и хронический синусит [25].

SPINK1-ассоциированный семейный панкреаmum. Случаи заболевания у пациентов с двумя патогенными вариантами гена SPINK1 в трансположении также классифицируются как аутосомно-рецессивный панкреатит. Гетерозиготное носительство патогенных вариантов гена SPINK1 может в значительной степени увеличивать риск развития панкреатита и вносить свой вклад 
в развитие ХП в рамках сложного мультигенного взаимодействия.

Варианты хронического панкреатита со сложным наследованием представляют собой наиболее важную категорию для всех типов панкреатита и других заболеваний ПЖ. Тщательное определение отдельных генетических факторов риска у пациента в конечном итоге способствует лучшему пониманию молекулярного патогенеза заболевания и персонализированному подходу в лечении больного в контексте парадигмы прецизионной медицины. Эта категория фокусируется на генетических вариантах, которые повышают восприимчивость к повреждению ПЖ через трипсинзависимый путь, неправильную укладку (мисфолдинг) белка, связанную с эндоплазматической сетью и активацией системы ответа на мисфолдинг, или другие механизмы ответа ацинарных или протоковых клеток на стресс или повреждение, включая нарушение регуляции кальциевого обмена. В эту категорию должны быть включены только генные варианты с доказанным патогенным эффектом или рассматриваемые в качестве вероятно патогенных вариантов (www.pancreasgenetics.org). Вместе с тем следует отдельно сохранять результаты генетического исследования пациента, так как у него могут быть выявлены варианты, не описанные в мировой литературе и геномных базах данных, однако, возможно, имеющие значение для развития заболевания.

В категорию «Варианты со сложным наследованием» должны быть включены патогенные или вероятно патогенные варианты генов SPINK1 и CTRC, обнаруженные в гетерозиготной форме. Варианты гена CFTR в этой категории отражаются, в случае если выявлен один генный вариант в гене или несколько патогенных вариантов в одной копии гена (то есть в цис-положении), при этом информация о нарушении функции белка-продукта гена отсутствует (например, потовая проба не проводилась) либо функция белка сохранена (например, уровни хлорида потовой пробы < 30 ммоль/л) [1].

Подгруппа «Другое» предназначена для генетических вариантов, не перечисленных выше, но считающихся генетическими факторами риска развития панкреатита.

Гень-модификаторь

Такие гены отличаются от генетических факторов предрасположенности тем, что изолированно не вызывают РОП или ХП, но фенотипически утяжеляют течение заболевания. К их числу в настоящей версии классификации отнесены мутации генов CLDN2 (ассоциация с приемом алкоголя), SLC26A9 (связь с тяжестью течения муковисцидоза и недостаточным ответом на терапию), GGT1 (ассоциация с панкреатитом и раком ПЖ), а также связанные с III группой крови (риск развития панкреатита и рака ПЖ) [1].

Категория «Другое» предназначена для генетических вариантов, не перечисленных выше, но считающихся значимыми в отношении модификации течения заболевания.

\section{Гипертриглииеридемические наследственные} синдромы

ГТГ отмечена в разделе «Токсико-метаболические факторы, гипертриглицеридемия». Однако наследственный генез ГТГ требует отражения и в этой части классификации для описания вариантов генов, наиболее часто связанных с развитием семейной хиломикронемии (гены липопротеинлипазы LPL и аполипопротеина C II APOC2) и другими менее распространенными вариантами мутаций одиночных генов или сложными комбинациями вариантов [28].

Многофакторный синдром хиломикронемии включает как генетические факторы, так и кофакторы окружающей среды в их сложных комбинациях. Эту категорию следует отмечать у пациентов с ГТГ после получения результатов генетического тестирования или при наличии у пациента убедительных клинических данных о фенотипе ГТГ на фоне известного существенного унаследованного генетического риска развития данного состояния.

В категорию «Другое» относят варианты, отмеченные в медицинской документации больного, но не попадающие в вышеупомянутые разделы. Например, сюда можно включить генные варианты ANGPTL3, APOA5, APOB, CELSR2, FABP4, FADS1, -2, -3, GCKR, GPIHBP1, LMF1, MLXIPL, $P P A R G$ и др. $[1,28]$.

Редкие неопухолевые генетически обусловленные нарушения поджелудочной железы

Данная категория - новая, она включает в себя несколько клинических синдромов нарушения функций ПЖ, которые могут быть вызваны патогенными вариантами в одном или нескольких генах.

Синдром Швахмана - Даймонда представляет собой редкое аутосомно-рецессивное заболевание, связанное с мутациями в гене $S B D S$ и, вероятно, других генах, таких как DNAJC21, ELF1 и SRP54, характеризуется развитием тяжелой 
ЭНПЖ без панкреатита, гематологическими нарушениями (например, циклическая нейтропения), низким ростом и дефектами опорно-двигательного аппарата [1, 29].

Синдром Йохансона - Близзарда - редкий аутосомно-рецессивный синдром, связанный с мутациями в гене убиквитинлигазы E3 (UBR1), характеризующийся липоматозом ПЖ с развитием ЭНПЖ, типичными чертами лица, аномалиями зубов, гипотиреозом, нейросенсорной тугоухостью, дефектами скальпа, урогенитальными и аноректальными аномалиями, низким ростом и когнитивными нарушениями различной степени [1].

Митохондриальные заболевания. Для функционирования ПЖ требуется большое количество энергии. Особое значение в регуляции обмена кальция имеет аденозинтрифосфат, его дефицит может предрасполагать к активации трипсина. Нарушение функции митохондрий увеличивает риск панкреатита [28]. К этой категории можно, например, отнести синдром Пирсона, характеризующийся прогрессирующим поражением различных систем органов и приводящий к ранней гибели больного. В эту же категорию следует включать и другие мутации, приводящие к развитию митохондриальной патологии, у пациентов с РОП или ХП [1].

Другое. Тропический панкреатит - исторический термин, используемый для описания идиопатического ХП в тропических регионах. Во многих случаях у этих пациентов присутствуют сложные генетические нарушения, схожие с другими сложно наследуемыми случаями ХП. Фенотипически выделяют две разновидности: «тропический кальцифицирующий панкреатит» и «фиброкалькулезный панкреатогенный диабет». Для того чтобы определить, представляют ли они собой различные синдромы, которые отличаются от других сложно наследуемых случаев панкреатита, и выявить факторы риска развития данной категории заболевания, необходимы дальнейшие исследования [30, 31].

\section{Аутоиммунный панкреатит}

АИП - воспалительное заболевание ПЖ с атипичным ответом В-лимфоцитов и эффектом применения кортикостероидов. Рассматриваются два основных типа.

АИП І типа патоморфологически описывают как лимфоплазмоцитарный склерозирующий панкреатит, который обычно связан с повышением уровня IgG4 и может быть частью IgG4ассоциированного заболевания с поражением других органов [28].
АИП II типа патоморфологически характеризуется гранулоцитарной деструкцией протокового эпителия. В настоящее время сывороточных маркеров АИП II типа не существует. Поскольку постановка точного диагноза с помощью биопсии ПЖ сопряжена с высоким риском, часто предпочтительным признается применение кортикостероидов ex juvantibus (пробное применение). В большом количестве наблюдений помимо АИП II типа у пациентов диагностируют воспалительные заболевания кишечника [1].

АИП неуточненный - сложные случаи панкреатита с очевидным аутоиммунным компонентом, не соответствующие критериям АИП I или II типа, но отвечающие на терапию кортикостероидами.

Рецидивирующий и тяжелый острый панкреатит К числу наиболее значимых факторов риска развития ХП относятся РОП и тяжелый ОП [13]. РОП устанавливают в случае второго и последующих эпизодов ОП независимо от степени тяжести, эта форма панкреатита считается гораздо более сильным фактором риска прогрессирования ХП, чем единственный эпизод ОП [13, 32].

Тяжелый ОП диагностируется при наличии синдрома персистирующей системной воспалительной реакции $\geq 48$ часов с полиорганной недостаточностью (дыхательная, сердечно-сосудистая и почечная), наличии показаний к пребыванию в отделении интенсивной терапии, хирургическим вмешательствам, а также в случае формирования постнекротических псевдокист.

К этиологическим факторам ОП/РОП, возникшим за пределами ацинарного и протокового аппарата ПЖ и не относящимся к токсико-метаболическим факторам, причисляют:

- билиарный ОП, который служит основным этиологическим фактором тяжелого ОП;

- панкреатит, развившийся после проведения эндоскопической ретроградной холангиопанкреатографии (постманипуляционный);

- инфекционные факторы - вирусные и другие в зависимости от результатов лабораторных исследований и выявленного возбудителя. Бактерии как этиологический фактор не следует путать со вторичной инфекцией при инфицированном панкреонекрозе.

Анализ информации о первом эпизоде ОП, РОП и ХП важен для понимания развития заболевания у отдельных пациентов, поскольку у некоторых из них путь к ХП выглядит как ОП $\rightarrow$ РОП $\rightarrow$ ХП, в то время как у других (примерно $40 \%)$ ХП развивается без РОП или ОП в анамнезе. 
Это позволяет предположить, что существует другой механизм патогенеза ХП. Важно фиксировать даты и осложнения каждого эпизода ОП, а также любые новые симптомы, в том числе СД, ЭНПЖ или изменение характера абдоминальной боли. Кроме того, должна быть описана частота приступов. Эти данные важны для оценки течения заболевания, результатов и эффективности выполненных вмешательств [32, 33].

\section{Обструктивный панкреатит}

Основное различие между первой и обсуждаемой версиями TIGAR-O - замена противоречивого термина «дисфункция сфинктера Одди» на «ампулярный стеноз» и расширение локализации и этиологии факторов, которые могут нарушать проходимость протоков ПЖ и способствовать развитию обструктивного ХП [1].

Патофизиологические механизмы образования кальцинатов в протоках ПЖ изучены плохо. Степень кальцификации/камнеобразования варьирует среди пациентов, поэтому рассматривается разделение пациентов на группы с кальцинатами преимущественно больших (вирсунголитиаз) и преимущественно малых (диффузная кальцификация) протоков [34]. Стриктуры главного панкреатического протока (ГПП) считаются значительными, если присутствует его расширение дистальнее места сужения. Новообразования также могут вызывать обструкцию ГПП с перманентным повреждением тканей ПЖ дистальнее места обструкции. Протоковая аденокарцинома ПЖ обычно инициирует десмопластическую реакцию, которая по своей сути не является ХП. В качестве причин обструктивного ХП следует рассматривать только локализованные протоковые аденокарциномы ПЖ с обструкцией ГПП и остаточным ХП после удаления опухоли. Внутрипротоковые папиллярно-муцинозные неоплазии с дистальным расширением ГПП из-за обструкции тоже могут рассматриваться в качестве причины обструктивного панкреатита. К «анатомическим вариантам» относятся периампулярные кисты двенадцатиперстной кишки, холедохоцеле, кисты санториниева протока (фокальная кистозная дилатация терминального отдела добавочного протока ПЖ), патология панкреатобилиарного конфлюенса, кольцевидная ПЖ и др. Pancreas divisum - распространенный вариант, служащий предметом исследований в настоящее время, - остается в отдельной категории [1].

\section{Обсуждение и заключение}

Наши знания о воспалительных заболеваниях ПЖ в последние десятилетия претерпели серьезные изменения, что во многом объясняется открытием генетических причин и многогранностью патогенеза панкреатитов [35]. Это формирует сложности восприятия клиницистами хронических заболеваний ПЖ и делает необходимым признание важности целостного подхода к профилактике и контролю заболеваний, требует от лечащего врача более глубокого понимания механизмов патогенеза, факторов риска/этиологии и конкретных биомаркеров активности и стадии заболевания $[1,2]$. Цель обновления классификации TIGAR-O состоит не столько в том, чтобы учесть недавно открытые и/или доказанные факторы риска, сколько в том, чтобы дать механистическое представление о различных заболеваниях ПЖ с общими характеристиками и лежащими в их основе механизмами. Например, ни атрофия, ни фиброз, ни ЭНПЖ, ни панкреатическая боль, ни СД не являются специфичными для ХП, особенно на ранних стадиях заболевания [36]. Дифференциально-диагностический ряд для выявления этих клинических признаков и биомаркеров включает АИП, синдромы, сопровождающиеся недостаточностью ПЖ, СД, образования, вызывающие обструкцию протоков ПЖ, внутрипротоковые папиллярно-муцинозные неоплазии, РПЖ и др. Кроме того, у пациента может наблюдаться более одного заболевания, что затрудняет диагностику и лечение на основании клинической картины и рутинных методов исследования. Таким образом, выявление и классификация факторов риска и этиологических факторов на основе результатов дальнейших исследований и установленных критериев позволяет ранжировать и подтверждать относительную вероятность заболеваний из дифференциально-диагностического ряда [37].

У пациентов с идиопатическим панкреатитом следует рассматривать все факторы риска. Историю болезни необходимо дополнять новой информацией (например, результатами генетического исследования) или изменениями в статусе факторов риска (например, прекращение курения, лечение ГТГ и устранение обструкции), датируя все изменения и дополнения.

Профилактика развития и прогрессирования заболевания в большей степени, чем поддерживающее лечение, будет зависеть от использования в здравоохранении новых информационных технологий, связывающих личность пациента, результаты его обследования, работающих с ним специалистов и мир информации. Кроме того, этот инструмент предоставит клиницисту, нацеленному на пациент-ориентированную помощь, 
осязаемую информацию для работы с пациентами. У них появится возможность обсуждать этиологию и факторы риска, а также способы непосредственного снижения индивидуальных рисков пациента [1].

Преимущество стандартизированного подхода к заболеваниям ПЖ на ранних стадиях заключается в обеспечении критического анализа в парадигме прецизионной медицины, необходимого для эффективного лечения заболевания. Обновленная этиологическая классификация панкреатитов TIGAR-O V2 имеет большое значение в определении модифицируемых факторов риска и профилактике их воздействия, что поможет предотвратить прогрессирование патологии ПЖ у пациентов, а также у членов их семей при выявлении генетических аспектов и генетическом консультировании. (ङ)

\section{Дополнительная информация}

\section{Финансирование}

Работа проведена без привлечения дополнительного финансирования со стороны третьих лиц.

\section{Конфликт интересов}

Авторы декларируют отсутствие явных и потенциальных конфликтов интересов, связанных с публикацией настоящей статьи.

\section{Участие авторов}

Все авторы внесли существенный вклад в проведение исследования и подготовку статьи, прочли и одобрили финальную версию перед публикацией.

\section{Литература}

1. Whitcomb DC; North American Pancreatitis Study Group. Pancreatitis: TIGAR-O Version 2 Risk/Etiology Checklist With Topic Reviews, Updates, and Use Primers. Clin Transl Gastroenterol. 2019;10(6):e00027. doi: 10.14309/ ctg.0000000000000027.

2. Petrov MS, Yadav D. Global epidemiology and holistic prevention of pancreatitis. Nat Rev Gastroenterol Hepatol. 2019;16(3):175-84. doi: 10.1038/s41575-018-0087-5.

3. Ивашкин ВТ, Маев ИВ, Охлобыстин АВ, Кучерявый ЮА, Трухманов АС, Шептулин АА, Шифрин ОС, Лапина ТЛ, Осипенко МФ, Симаненков ВИ, Хлынов ИБ, Алексеенко СА, Алексеева ОП, Чикунова МВ. Рекомендации Российской гастроэнтерологической ассоциации по диагностике и лечению хронического панкреатита. Российский журнал гастроэнтерологии, гепатологии, колопроктологии. 2014;24(4):70-97.

4. Forsmark CE, Andersen DK, Farrar JT, Golden M, Habtezion A, Husain SZ, Li L, Mayerle J, Pandol SJ, Uc A, Zhu Z, Yadav D. Accelerating the Drug Delivery Pipeline for Acute and Chronic Pancreatitis: Summary of the Working Group on Drug Development and Trials in Chronic Pancreatitis at the National Institute of Diabetes and Digestive and Kidney Diseases Workshop. Pancreas. 2018;47(10):1200-7. doi: 10.1097/MPA.0000000000001174.

5. Etemad B, Whitcomb DC. Chronic pancreatitis: diagnosis, classification, and new genetic developments. Gastroenterology. 2001;120(3): 682-707. doi: 10.1053/gast.2001.22586.

6 . Whitcomb DC. What is personalized medicine and what should it replace? Nat Rev Gastroenterol Hepatol. 2012;9(7):418-24. doi: 10.1038/ nrgastro.2012.100.

7. Хатьков ИЕ, Маев ИВ, Бордин ДС, Кучерявый ЮА, Абдулхаков СР, Алексеенко СА, Алиева ЭИ, Алиханов РБ, Арутюнов ГГ, Бакулин ИГ, Барановский АЮ, Белобородо-

ва ЕВ, Белоусова ЕА, Буриев ИМ, Быстровская ЕВ, Вертянкин СВ, Винокурова ЛВ, Гальперин ЭИ, Горелов АВ, Гриневич ВБ, Данилов МВ, Дарвин ВВ, Дубцова ЕА, Дюжева ТГ, Егоров ВИ, Ефанов МГ, Загайнов ВЕ, Захарова НВ, Израилов РЕ, Корочанская НВ, Корниенко ЕА, Коробка ВЛ, Коханенко НЮ, Ливзан МА, Лоранская ИД, Никольская КА, Осипенко МФ, Охлобыстин АВ, Пасечников ВД, Плотникова ЕЮ, Полякова СИ, Саблин ОА, Симаненков ВИ, Урсова НИ, Цвиркун ВВ, Цуканов ВВ, Шабунин АВ, Ивашкин ВТ. Российский консенсус по диагностике и лечению хронического панкреатита: этиология хронического панкреатита и диагностика внешнесекреторной недостаточности поджелудочной железы. Дневник казанской медицинской школы. 2017;2(16): 33-8.

8.Бордин ДС, Кучерявый ЮА. Ключевые позиции панъевропейских клинических рекомендаций по диагностике и лечению хронического панкреатита в фокусе гастроэнтеролога. РМЖ. 2017;25(10):730-7.

9. Кучерявый ЮА, Кирюкова МА, Дубцова ЕА, Бордин ДС. Клинические рекомендации ACG-2020 по диагностике и лечению хронического панкреатита: обзор ключевых положений в практическом преломлении. Эффективная фармакотерапия. 2020;16(15): 60-73. doi: 10.33978/2307-3586-2020-16-1560-72.

10.Schneider A, Löhr JM, Singer MV. The M-ANNHEIM classification of chronic pancreatitis: introduction of a unifying classification system based on a review of previous classifications of the disease. J Gastroenterol. 2007;42(2): 101-19. doi: 10.1007/s00535-006-1945-4.

11. Хатьков ИЕ, Маев ИВ, Абдулхаков СР, Алексеенко СА, Алиева ЭИ, Алиханов РБ, Бакулин ИГ, Барановский АЮ, Белобородова ЕВ, Белоусова ЕА, Буриев ИМ, Быстровская ЕВ,
Вертянкин СВ, Винокурова ЛВ, Гальперин ЭИ, Горелов АВ, Гриневич ВБ, Данилов МВ, Дарвин ВВ, Дубцова ЕА, Дюжева ТГ, Егоров ВИ, Ефанов МГ, Захарова НВ, Загайнов BЕ, Ивашкин ВТ, Израилов РЕ, Корочанская НВ, Корниенко ЕА, Коробка ВЛ, Коханенко НЮ, Кучерявый ЮА, Ливзан МА, Лоранская ИД, Никольская КА, Осипенко МФ, Охлобыстин АВ, Пасечников ВД, Плотникова ЕЮ, Полякова СИ, Саблин ОА, Симаненков ВИ, Урсова НИ, Цвиркун ВВ, Цуканов ВВ, Шабунин АВ, Бордин ДС. Российский консенсус по диагностике и лечению хронического панкреатита. Терапевтический архив. 2017;89(2):105-13. doi: 10.17116/ terarkh2017892105-113.

12. Whitcomb DC, Frulloni L, Garg P, Greer JB, Schneider A, Yadav D, Shimosegawa T. Chronic pancreatitis: An international draft consensus proposal for a new mechanistic definition. Pancreatology. 2016;16(2):218-24. doi: 10.1016/j.pan.2016.02.001.

13. Sankaran SJ, Xiao AY, Wu LM, Windsor JA, Forsmark CE, Petrov MS. Frequency of progression from acute to chronic pancreatitis and risk factors: a meta-analysis. Gastroenterology. 2015;149(6):1490-500.e1. doi: 10.1053/j.gastro.2015.07.066.

14. Bai HX, Giefer M, Patel M, Orabi Al, Husain SZ. The association of primary hyperparathyroidism with pancreatitis. J Clin Gastroenterol. 2012;46(8):656-61. doi: 10.1097/MCG. 0b013e31825c446c.

15. Pedersen SB, Langsted A, Nordestgaard BG. Nonfasting Mild-to-Moderate Hypertriglyceridemia and Risk of Acute Pancreatitis. JAMA Intern Med. 2016;176(12):1834-42. doi: 10.1001/ jamainternmed.2016.6875.

16. Rawla P, Sunkara T, Thandra KC, Gaduputi V. Hypertriglyceridemia-induced pancreatitis: updated review of current treatment and preventive strategies. Clin J Gastroenterol. 
2018;11(6):441-8. doi: 10.1007/s12328-0180881-1.

17. Chung EK, Lee JH, Jang DK, Lee SH, Lee JH, Park BJ, Kwon K, Lee JK. Causative Agents of Drug-Induced Pancreatitis: A Nationwide Assessment. Pancreas. 2018;47(10):1328-36. doi: 10.1097/MPA.0000000000001152.

18. Khurana V, Barkin JS. Pancreatitis induced by environmental toxins. Pancreas. 2001;22(1): 102-5. doi: 10.1097/00006676-20010100000019.

19. Тарасова ЖС, Бордин ДС, Килейников дВ, Кучерявый ЮА. Панкреатогенный сахарный диабет: взгляд эндокринолога и гастроэнтеролога. Эффективная фармакотерапия. 2020;16(15):92-101. doi: 10.33978/2307-35862020-16-15-92-100.

20. Setiawan VW, Pandol SJ, Porcel J, Wei PC, Wilkens LR, Le Marchand L, Pike MC, Monroe KR. Dietary Factors Reduce Risk of Acute Pancreatitis in a Large Multiethnic Cohort. Clin Gastroenterol Hepatol. 2017;15(2):257-65.e3. doi: 10.1016/j.cgh.2016.08.038.

21. Маев ИВ, Кучерявый ЮА, Андреев ДН. Ожирение и коморбидность: пособие для врачей. М.: Прима Принт, 2016.

22. Маев ИВ, Москалева АБ, Сальникова ЕА, Кучерявый ЮА. Ожирение и болезни поджелудочной железы. Consilium Medicum 2008;(1): 24-9.

23. Kucheryavyy YuA, Andreev DN. Nutritional status in patients with chronic pancreatitis. J Nutr Ther. 2014;(3):122-32. doi: 10.6000/19295634.2014.03.03.3.

24. Кучерявый ЮА, Маев ИВ, Петрова НВ, Смирнов АВ, Тибилова 3Ф. Хронический панкреатит в свете последних открытий в молекулярной генетике. Клинические перспективы гастроэнтерологии, гепатологии. 2012;(4): 3-10.

25. Farrell PM, White TB, Ren $C L$, Hempstead $S E$, Accurso F, Derichs N, Howenstine M, McColley SA, Rock M, Rosenfeld M, Sermet-Gaudelus I, Southern KW, Marshall BC, Sosnay PR. Diagnosis of Cystic Fibrosis: Consensus Guidelines from the Cystic Fibrosis Foundation. J Pediatr. 2017;181S:S4-15.e1. doi: 10.1016/j. jpeds.2016.09.064. Erratum in: J Pediatr. 2017;184:243.

\section{References}

1. Whitcomb DC; North American Pancreatitis Study Group. Pancreatitis: TIGAR-O Version 2 Risk/Etiology Checklist With Topic Reviews, Updates, and Use Primers. Clin Transl Gastroenterol. 2019;10(6):e00027. doi: 10.14309/ ctg.0000000000000027.

2. Petrov MS, Yadav D. Global epidemiology and holistic prevention of pancreatitis. Nat Rev Gastroenterol Hepatol. 2019;16(3):175-84. doi: 10.1038/s41575-018-0087-5.
26. De Boeck K, Amaral MD. Progress in therapies for cystic fibrosis. Lancet Respir Med. 2016;4(8): 662-74. doi: 10.1016/S2213-2600(16)00023-0.

27. Moulin $P$, Dufour R, Averna M, Arca M, Cefalù $A B$, Noto $D, D^{\prime}$ Erasmo $L$, Di Costanzo $A$, Marçais C, Alvarez-Sala Walther LA, Banach M, Borén J, Cramb R, Gouni-Berthold I, Hughes E, Johnson C, Pintó X, Reiner Ž, van Lennep JR, Soran H, Stefanutti C, Stroes E, Bruckert E. Identification and diagnosis of patients with familial chylomicronaemia syndrome (FCS): Expert panel recommendations and proposal of an "FCS score". Atherosclerosis. 2018;275:265-72. doi: 10.1016/j.atherosclerosis.2018.06.814.

28. Литвинова МM, Хафизов КФ, Сперанская АС, Мацвай АД, Никольская КА, Винокурова ЛВ, Дубцова ЕА, Мухина ТФ, Хавкин АИ, Бордин ДС. Спектр мутаций гена CFTR у больных хроническим панкреатитом в России. Вопросы детской диетологии. 2020;18(3):5-18. doi: 10.20953/1727-57842020-3-5-18.

29. Biczo G, Vegh ET, Shalbueva N, Mareninova OA, Elperin J, Lotshaw E, Gretler S, Lugea A, Malla SR, Dawson D, Ruchala P, Whitelegge J, French SW, Wen L, Husain SZ, Gorelick FS, Hegyi P, Rakonczay Z Jr, Gukovsky I, Gukovskaya AS. Mitochondrial Dysfunction, Through Impaired Autophagy, Leads to Endoplasmic Reticulum Stress, Deregulated Lipid Metabolism, and Pancreatitis in Animal Models. Gastroenterology. 2018;154(3):689-703. doi: 10.1053/j.gastro.2017.10.012.

30. Ипатова МГ, Деордиева ЕА, Швец ОА, Мухина АА, Моисеева АА, Родина ЮА, Шумилов ПВ, Павлова АВ, Райкина ЕВ, Асанов АЮ, Литвинова ММ, Щербина АЮ. Генетические и клинико-лабораторные особенности синдрома Швахмана-Даймонда в России: проспективное исследование. Вопросы современной педиатрии. 2019;18(5):393-400. doi: 10.15690/vsp.v18i5.2057.

31. Singh G, Bhat B, Jayadev MSK, Madhusudhan C, Singh A. mutTCPdb: a comprehensive database for genomic variants of a tropical country neglected disease-tropical calcific pancreatitis. Database (Oxford). 2018;2018:bay043. doi: 10.1093/database/bay043.

32. Буеверов АО, Кучерявый ЮА. IgG4-ассоциированная болезнь: монография. М.: Форте Принт; 2014. 128 с.

3. Ivashkin VT, Maev IV, Okhlobystin AV, Kucheryavyy YuA, Trukhmanov AS, Sheptulin AA, Shifrin OS, Lapina TL, Osipenko MF, Simanenkov VI, Khlynov IB, Alekseyenko SA, Alekseyeva OP, Chikunova M.V. [Guidelines of the Russian gastroenterological association on diagnostics and treatment of a chronic pancreatitis]. Russian Journal of Gastroenterology, Hepatology and Coloproctology. 2014;24(4): 70-97. Russian.
33. Guda NM, Muddana V, Whitcomb DC, Levy P, Garg P, Cote G, Uc A, Varadarajulu S, Vege SS, Chari ST, Forsmark CE, Yadav D, Reddy DN, Tenner S, Johnson CD, Akisik F, Saluja AK, Lerch MM, Mallery JS, Freeman ML. Recurrent Acute Pancreatitis: International State-of-the-Science Conference With Recommendations. Pancreas. 2018;47(6):653-66. doi: 10.1097/MPA.0000000000001053.

34. Coté GA, Yadav D, Abberbock JA, Whitcomb DC, Sherman S, Sandhu BS, Anderson MA, Lewis MD, Alkaade S, Singh VK, Baillie J, Banks PA, Conwell D, Guda NM, Muniraj T, Tang G, Brand R, Gelrud A, Amann ST, Forsmark CE, Wilcox MC, Slivka A, Gardner TB. Recurrent Acute Pancreatitis Significantly Reduces Quality of Life Even in the Absence of Overt Chronic Pancreatitis. Am J Gastroenterol. 2018;113(6):906-12. doi: 10.1038/s41395018-0087-7.

35. Маев ИВ, Кучерявый ЮА. Литостатин: современный взгляд на биологическую роль и патогенез хронического панкреатита. Российский журнал гастроэнтерологии, гепатологии, колопроктологии. 2006;16(5):4-10.

36. Литвинова ММ, Хафизов КФ, Шипулин ГА, Айгинин АА, Винокурова ЛВ, Никольская КА, Дубцова ЕА, Бордин ДС, Асанов АЮ. Генетические факторы развития хронического панкреатита. Вопросы практической педиатрии. 2018;13(3):29-40. doi: 10.20953/18177646-2018-3-29-40.

37. Whitcomb DC, Shimosegawa T, Chari ST, Forsmark CE, Frulloni L, Garg P, Hegyi P, Hirooka $Y$, Irisawa A, Ishikawa $T$, Isaji $S$, Lerch MM, Levy P, Masamune A, Wilcox CM, Windsor J, Yadav D, Sheel A, Neoptolemos JP; Working Group for the International (IAP - APA - JPS EPC) Consensus Guidelines for Chronic Pancreatitis. International consensus statements on early chronic Pancreatitis. Recommendations from the working group for the international consensus guidelines for chronic pancreatitis in collaboration with The International Association of Pancreatology, American Pancreatic Association, Japan Pancreas Society, PancreasFest Working Group and European Pancreatic Club. Pancreatology. 2018;S1424-3903(18)30113-3. doi: 10.1016/j. pan.2018.05.008.

4. Forsmark $C E$, Andersen DK, Farrar JT, Golden M, Habtezion A, Husain SZ, Li L, Mayerle J, Pandol SJ, Uc A, Zhu Z, Yadav D. Accelerating the Drug Delivery Pipeline for Acute and Chronic Pancreatitis: Summary of the Working Group on Drug Development and Trials in Chronic Pancreatitis at the National Institute of Diabetes and Digestive and Kidney Diseases Workshop. Pancreas. 2018;47(10):1200-7. doi: 10.1097/MPA.0000000000001174. 
5. Etemad B, Whitcomb DC. Chronic pancreatitis: diagnosis, classification, and new genetic developments. Gastroenterology. 2001;120(3): 682-707. doi: 10.1053/gast.2001.22586.

6. Whitcomb DC. What is personalized medicine and what should it replace? Nat Rev Gastroenterol Hepatol. 2012;9(7):418-24. doi: 10.1038/ nrgastro.2012.100.

7. Khatkov IE, Mayev IV, Bordin DS, Kucheryavy YuA, Abdulkhakov SR, Alekseenko SA, Alieva El, Alikhanov RB, Arutyunov GG, Bakulin IG, Baranovsky AYu, Beloborodova EV, Belousova EA, Buriev IM, Bystrovskaya EV, Vertyankin SV, Vinokurova LV, Galperin El, Gorelov AV, Grinevich VB, Danilov MV, Darvin VV, Dubtsova EA, Dyuzheva TG, Egorov VI, Efanov MG, Zagaynov VE, Zakharova NV, Izrailov RE, Korochanskaya NV, Kornienko EA, Korobka VL, Kokhanenko NYu, Livzan MA, Loranskaya ID, Nikolskaya KA, Osipenko MF, Okhlobystin AV, Pasechnikov VD, Plotnikova EYu, Polyakova SI, Sablin OA, Simanenkov VI, Ursova NI, Tsvirkun VV, Tsukanov VV, Shabunin AV, Ivashkin VT. Russian consensus on the diagnosis and treatment of chronic pancreatitis: etiology of chronic pancreatitis and diagnosis of exocrine pancreatic insufficiency. Kazan Medical School Diary. 2017;2(16):33-8. Russian.

8. Bordin DS, Kucheryavy YuA. [The key points of the pan-European clinical guidelines for the diagnosis and treatment of chronic pancreatitis in the focus of gastroenterologist]. Russian Medical Journal. 2017;25(10):730-7. Russian.

9. Kucheryavy YuA, Kiryukova MA, Dubtsova YeA, Bordin DS. [ACG-2020 Clinical Guidelines for the Diagnosis and Treatment of Chronic Pancreatitis: Overview of Key Provisions in Practice]. Effektivnaya farmakoterapiya [Effective Pharmacotherapy]. 2020;16(15):60-73. Russian. doi: 10.33978/2307-3586-2020-16-15-6072.

10. Schneider A, Löhr JM, Singer MV. The M-ANNHEIM classification of chronic pancreatitis: introduction of a unifying classification system based on a review of previous classifications of the disease. J Gastroenterol. 2007;42(2): 101-19. doi: 10.1007/s00535-006-1945-4.

11. Khatkov IE, Maev IV, Abdulkhakov SR, Alekseenko SA, Alieva El, Alikhanov RB, Bakulin IG, Baranovsky AYu, Beloborodova EV, Belousova EA, Buriev IM, Bystrovskaya EV, Vertyankin SV, Vinokurova LV, Galperin El, Gorelov AV, Grinevich VB, Danilov MV, Darvin VV, Dubtsova EA, Dyuzheva TG, Egorov VI, Efanov MG, Zakharova NV, Zagainov VE, Ivashkin VT, Izrailov RE, Korochanskaya NV, Kornienko EA, Korobka VL, Kokhanenko NYu, Kucheryavy YuA, Livzan MA, Loranskaya ID, Nikolskaya KA, Osipenko MF, Okhlobystin AV, Pasechnikov VD, Plotnikova EYu, Polyakova SI, Sablin OA, Simanenkov VI, Ursova NI, Tsvirkun VV, Tsukanov VV, Shabunin AV, Bordin DS. [The Russian consensus on the diagnosis and treatment of chronic pancreatitis]. Therapeutic Archive. 2017;89(2):105-13. Russian. doi: 10.17116/terarkh2017892105-113.

12. Whitcomb DC, Frulloni L, Garg P, Greer JB, Schneider A, Yadav D, Shimosegawa T. Chronic pancreatitis: An international draft consensus proposal for a new mechanistic definition. Pancreatology. 2016;16(2):218-24. doi: 10.1016/j.pan.2016.02.001.

13. Sankaran SJ, Xiao AY, Wu LM, Windsor JA, Forsmark CE, Petrov MS. Frequency of progression from acute to chronic pancreatitis and risk factors: a meta-analysis. Gastroenterology. 2015;149(6):1490-500.e1. doi: 10.1053/j.gastro.2015.07.066.

14. Bai HX, Giefer M, Patel M, Orabi Al, Husain SZ. The association of primary hyperparathyroidism with pancreatitis. J Clin Gastroenterol. 2012;46(8):656-61. doi: 10.1097/MCG. Ob013e31825c446c.

15. Pedersen SB, Langsted A, Nordestgaard BG. Nonfasting Mild-to-Moderate Hypertriglyceridemia and Risk of Acute Pancreatitis. JAMA Intern Med. 2016;176(12):1834-42. doi: 10.1001/ jamainternmed.2016.6875.

16. Rawla P, Sunkara T, Thandra KC, Gaduputi V. Hypertriglyceridemia-induced pancreatitis: updated review of current treatment and preventive strategies. Clin J Gastroenterol. 2018;11(6): 441-8. doi: 10.1007/s12328-018-0881-1.

17. Chung EK, Lee JH, Jang DK, Lee SH, Lee JH, Park BJ, Kwon K, Lee JK. Causative Agents of Drug-Induced Pancreatitis: A Nationwide Assessment. Pancreas. 2018;47(10):1328-36. doi: 10.1097/MPA.0000000000001152.

18. Khurana V, Barkin JS. Pancreatitis induced by environmental toxins. Pancreas. 2001;22(1): 102-5. doi: 10.1097/00006676-20010100000019.

19. Tarasova ZhS, Bordin DS, Kileynikov DV, Kucheryavy YuA. [Pancreatogenic Diabetes Mellitus: Endocrinologist's and Gastroenterologist's Point of View]. Effektivnaya farmakoterapiya [Effective Pharmacotherapy]. 2020;16(15):92101. Russian. doi: 10.33978/2307-3586-202016-15-92-100.

20. Setiawan VW, Pandol SJ, Porcel J, Wei PC, Wilkens LR, Le Marchand L, Pike MC, Monroe KR. Dietary Factors Reduce Risk of Acute Pancreatitis in a Large Multiethnic Cohort. Clin Gastroenterol Hepatol. 2017;15(2):257-65.e3. doi: 10.1016/j.cgh.2016.08.038.

21. Maev IV, Kucheryavyy YuA, Andreev DN. [Obesity and comorbidity: a manual for doctors]. Moscow: Prima Print, 2016. Russian.

22. Maev IV, Moskalyova AB, Salnikova EA, Kucheryavyy YuA. [Obesity and pancreatic diseases]. Consilium Medicum. 2008;(1):24-9. Russian.

23. Kucheryavyy YuA, Andreev DN. Nutritional status in patients with chronic pancreatitis. J Nutr Ther. 2014;(3):122-32. doi: 10.6000/19295634.2014.03.03.3.
24. Kucheryavy YuA, Mayev IV, Petrova NV, Smirnov AV, Tibilova ZF. [Chronic pancreatitis in the light of recent molecular genetics discoverings]. Klinicheskie perspektivy gastroenterologii, gepatologii [Clinical Perspectives in Gastroenterology, Hepatology]. 2012;(4):3-10. Russian.

25. Farrell PM, White TB, Ren CL, Hempstead $S E$, Accurso F, Derichs N, Howenstine M, McColley $S A$, Rock M, Rosenfeld M, Sermet-Gaudelus I, Southern KW, Marshall BC, Sosnay PR. Diagnosis of Cystic Fibrosis: Consensus Guidelines from the Cystic Fibrosis Foundation. J Pediatr. 2017;181S:S4-15.e1. doi: 10.1016/j. jpeds.2016.09.064. Erratum in: J Pediatr. 2017; 184:243.

26. De Boeck K, Amaral MD. Progress in therapies for cystic fibrosis. Lancet Respir Med. 2016;4(8): 662-74. doi: 10.1016/S2213-2600(16)00023-0.

27. Moulin $P$, Dufour R, Averna M, Arca M, Cefalù $A B$, Noto $D$, D'Erasmo $L$, Di Costanzo $A$, Marçais C, Alvarez-Sala Walther LA, Banach M, Borén J, Cramb R, Gouni-Berthold I, Hughes E, Johnson C, Pintó X, Reiner Ž, van Lennep JR, Soran H, Stefanutti C, Stroes E, Bruckert E. Identification and diagnosis of patients with familial chylomicronaemia syndrome (FCS): Expert panel recommendations and proposal of an "FCS score". Atherosclerosis. 2018;275:265-72. doi: 10.1016/j.atherosclerosis.2018.06.814.

28. Litvinova MM, Khafizov KF, Speranskaya AS, Matsvay AD, Nikolskaya KA, Vinokurova LV, Dubtsova EA, Muhina TF, Khavkin Al, Bordin DS. [Spectrum of CFTR gene mutations in patients with chronic pancreatitis in Russia]. Pediatric Nutrition. 2020;18(3):5-18. Russian. doi: 10.20953/1727-5784-2020-3-5-18.

29. Biczo G, Vegh ET, Shalbueva N, Mareninova OA, Elperin J, Lotshaw E, Gretler S, Lugea $A$, Malla SR, Dawson D, Ruchala P, Whitelegge J, French SW, Wen L, Husain SZ, Gorelick FS, Hegyi P, Rakonczay Z Jr, Gukovsky I, Gukovskaya AS. Mitochondrial Dysfunction, Through Impaired Autophagy, Leads to Endoplasmic Reticulum Stress, Deregulated Lipid Metabolism, and Pancreatitis in Animal Models. Gastroenterology. 2018;154(3):689-703. doi: 10.1053/j. gastro.2017.10.012.

30. Ipatova MG, Deordieva EA, Shvets OA, Mukhina AA, Moiseeva AA, Rodina YuA, Shumilov PV, Pavlova AV, Raikina EV, Asanov AYu, Litvinova MM, Shcherbina AY. [Genetic and Clinical Features of Shwachman-Diamond Syndrome in Russian Population: Prospective Study]. Voprosy sovremennoy pediatrii [Current Pediatrics]. 2019;18(5):393-400. Russian. doi: 10.15690/vsp.v18i5.2057.

31. Singh G, Bhat B, Jayadev MSK, Madhusudhan C, Singh A. mutTCPdb: a comprehensive database for genomic variants of a tropical country neglected disease-tropical calcific pancreatitis. Database (Oxford). 2018;2018:bay043. doi: 10.1093/database/bay043. 
32. Bueverov AO, Kucheryavyy YuA. [lgG4-related disease]. Moscow: Forte Print; 2014. 128 p. Russian.

33. Guda NM, Muddana V, Whitcomb DC, Levy P, Garg P, Cote G, Uc A, Varadarajulu S, Vege SS, Chari ST, Forsmark CE, Yadav D, Reddy DN, Tenner S, Johnson CD, Akisik F, Saluja AK, Lerch MM, Mallery JS, Freeman ML. Recurrent Acute Pancreatitis: International State-of-the-Science Conference With Recommendations. Pancreas. 2018;47(6):653-66. doi: 10.1097/MPA.0000000000001053.

34. Coté GA, Yadav D, Abberbock JA, Whitcomb DC, Sherman S, Sandhu BS, Anderson $M A$, Lewis $M D$, Alkaade $S$, Singh VK, Baillie J, Banks PA, Conwell D, Guda NM, Muniraj T, Tang G, Brand R, Gelrud A, Amann ST, Forsmark CE, Wilcox MC, Slivka A, Gardner TB.
Recurrent Acute Pancreatitis Significantly Reduces Quality of Life Even in the Absence of Overt Chronic Pancreatitis. Am J Gastroenterol. 2018;113(6):906-12. doi: 10.1038/s41395018-0087-7.

35. Maev IV, Kucheryavyy YuA. [Lithostatin: a modern view on biological role and pathogenesis of chronic pancreatitis]. Russian Journal of Gastroenterology, Hepatology, Coloproctology. 2006;16(5):4-10. Russian.

36. Litvinova MM, Khafizov KF, Shipulin GA, Ayginin AA, Vinokurova LV, Nikolskaya KA, Dubtsova EA, Bordin DS, Asanov AYu. [Genetic factors of the development of chronic pancreatitis]. Clinical Practice in Pediatrics. 2018;13(3):2940. Russian. doi: 10.20953/1817-7646-2018-329-40.

\title{
The revised pancreatitis etiology-based classification system TIGAR-0, version 2: adaptation for the Russian clinical practice
}

\author{
D.S. Bordin'1,2,3 • Yu.A. Kucheryavyy ${ }^{2,4}$ • M.A. Kiryukova ${ }^{1}$
}

The discoveries in molecular genetics and breakthrough visualization techniques in the last 20 years have changed our understanding of the pancreatitis causes and biomarkers, expanded our knowledge on the pathophysiology of the disease, and promoted the development of new additional conservative treatments. From the practical perspective, the physician's comprehension of the etiology is of particular importance. It is for this reason that the activities to elaborate an etiology-based classification of pancreatitis have been already started since long ago. The first internationally acknowledged system was TIGAR-O checklist, introduced in 2001. Being innovative at the time, it structured our understanding of the etiology of chronic pancreatitis. The revised version (version 2) was published in 2019 and is less known to the Russian medical community, although from the authors' point of view, it has been substantially extended and structured to be maximally convenient and useful for physicians in routine medical practice. The review presents key provisions of the TIGAR-O, version 2 and recommendations for its adaption to the Russian clinical setting.

Key words: chronic pancreatitis, acute pancreatitis, classification, diagnostics, genetic factors, exocrine pancreatic insufficiency, diabetes mellitus

For citation: Bordin DS, Kucheryavyy YuA, Kiryukova MA. The revised pancreatitis etiology-based classification system TIGAR-O, version 2: adaptation for the Russian clinical practice. Almanac of Clinical Medicine. 2020;48(6):349-63. doi: 10.18786/2072-0505-2020-48-062.

Received 19 August 2020; revised 17 November 2020; accepted 19 November 2020; published online 10 December 2020

\section{Conflict of interests}

The authors declare that they have no conflict of interests.

All the authors made their significant contributions to the research and preparation of the article, have read and approved the final version before submission.

37. Whitcomb DC, Shimosegawa T, Chari ST, Forsmark CE, Frulloni L, Garg P, Hegyi P, Hirooka $Y$, Irisawa A, Ishikawa T, Isaji $S$, Lerch MM, Levy P, Masamune A, Wilcox CM, Windsor J, Yadav D, Sheel A, Neoptolemos JP; Working Group for the International (IAP - APA - JPS EPC) Consensus Guidelines for Chronic Pancreatitis. International consensus statements on early chronic Pancreatitis. Recommendations from the working group for the international consensus guidelines for chronic pancreatitis in collaboration with The International Association of Pancreatology, American Pancreatic Association, Japan Pancreas Society, PancreasFest Working Group and European Pancreatic Club. Pancreatology. 2018;S1424-3903(18)30113-3. doi: 10.1016/j. pan.2018.05.008

Dmitry S. Bordin - MD, PhD, Head of Department of Pancreatic, Biliary, and Upper Digestive tract disorders'; Professor, Chair of Propaedeutics of Internal Diseases and Gastroenterology2; Professor, Chair of Outpatient Therapy and Family Medicine, Faculty of Postgraduate Education ${ }^{3}$; ORCID: https:// orcid.org/0000-0003-2815-3992. Tel.: +7 (495) 30430 35, ext. 1585. E-mail: d.bordin@mknc.ru

Yury A. Kucheryavyy - MD, PhD, Associate Professor, Chair of Propaedeutics of Internal Diseases and Gastroenterology2; Head of Department of Gastroenterology ${ }^{4}$; ORCID: https://orcid.org/00000001-7760-2091

$\triangle$ 2/2 Rublevskoe predmest'e ul., derevnya Glukhovo, Krasnogorsk, Moskovskaya oblast', 143421, Russian Federation. Tel.: +7 (495) 6453377. E-mail: proped@mail.ru

Maria A. Kiryukova - MD, Junior Research Fellow Department of Pancreatic and Biliary Diseases'; ORCID: https://orcid.org/0000-0002-6946-3826. E-mail:m.kirukova@mknc.ru

The Loginov Moscow Clinical Scientific Center 86 Entuziastov shosse, Moscow, 111123, Russian Federation

${ }^{2}$ A.I. Yevdokimov Moscow State University of Medicine and Dentistry; 20-1 Delegatskaya ul., Moscow, 127473, Russian Federation

${ }^{3}$ Tver State Medical University; 4 Sovetskaya ul., Tver, 170100, Russian Federation

${ }^{4}$ Il'inskaya Hospital; 2/2 Rublevskoe predmest'e ul., derevnya Glukhovo, Krasnogorsk, Moskovskaya oblast', 143421, Russian Federation 